\section{SAT0239 PREDICTION OF LONG-TERM EVOLUTIONARY PROFILES IN EOSINOPHILIC GRANULOMATOSIS WITH POLYANGIITIS (CHURG-STRAUSS) BASED ON BASELINE AND FOLLOW-UP CHARACTERISTICS}

Matthias Papo ${ }^{1}$, Renato A. Sinico ${ }^{2}$, Vítor Teixeira ${ }^{3}$, Maria-Letizia Urban ${ }^{4}$ Juliane Mahrhold ${ }^{5}$, Sara Monti ${ }^{6}$, Giulia Cassone ${ }^{7}$, Franco Schiavon ${ }^{8}$, Benjamin Seeliger ${ }^{9}$, Thomas Neumann ${ }^{10}$, Claus Kroegel ${ }^{11}$, Matthieu Groh ${ }^{12}$ Chiara Marvisi ${ }^{13}$, Maxime Samson ${ }^{14}$, Thomas Barba ${ }^{15}$, David Jayne ${ }^{3}$, Bernhard Hellmich ${ }^{5}$, Carlomaurizio Montecucco ${ }^{6}$, Carlo Salvarani ${ }^{7}$, JeanEmmanuel Kahn ${ }^{12}$, Bernard Bonnotte ${ }^{14}$, Cécile-Audrey Durel ${ }^{15}$, Luc Mouthon ${ }^{1}$, Xavier Puéchal ${ }^{1}$, Loïc Guillevin ${ }^{1}$, Giacomo Emmi ${ }^{4}$, Augusto Vaglio ${ }^{13}$, Benjamin Terrier', French Vasculitis Study Group and EGPA European Collaborative Initiative. ${ }^{1}$ Internal Medicine, Paris, France; ${ }^{2}$ Medicine and Surgery, Milan, Italy, ${ }^{3}$ Medicine, Cambridge, United Kingdom; ${ }^{4}$ Experimental and Clinical Medicine, Florence, Italy; Internal Medicine, Kirchheim, Germany; ${ }^{6}$ Rheumatology, Pavia, Italy, ${ }^{7}$ Rheumatology, Reggio Emilia and Modena, Italy; ${ }^{8}$ Rheumatology, Padova, Italy; ${ }^{9}$ Respiratory Medicine, Hannover, Germany; ${ }^{10}$ Rheumatology, St. Gallen, Switzerland; ${ }^{11}$ Pneumology, Jena, Germany, ${ }^{12}$ Internal Medicine, Suresnes, France; ${ }^{13}$ Nephrology, Parma, Italy, ${ }^{14}$ Internal Medicine, Dijon, France; ${ }^{15}$ Internal Medicine, Lyon, France

Background: Eosinophilic granulomatosis with polyangiitis (EGPA) (ChurgStrauss) is a small-vessel necrotizing vasculitis characterized by blood and tissue eosinophilia and asthma. Glucocorticoids (GCs) control the disease, but GC-dependence is frequent. Evolving concepts distinguish vasculitis-related symptoms from asthma and/or ENT manifestations. That distinction has become even more important since the development of Bcell and eosinophil-targeted therapies.

Objectives: This study aimed to describe and identify characteristics predicting long-term EGPA outcomes.

Methods: We set up a multicenter European cohort that included 636 EGPA patients. Based on recent consensus, we distinguished 4 EGPAevolutionary profiles: GC-dependent asthma and/or ENT manifestations (requiring prednisone $>7.5 \mathrm{mg} / \mathrm{d}$ ), $\geq 1$ vasculitis relapse(s) (excluding asthma and/or ENT flares), both phenotypes, and complete remission (no GC-dependent asthma/ENT signs, no vasculitis relapse and prednisone $<5 \mathrm{mg} / \mathrm{d}$ at last follow-up). Baseline and follow-up characteristics predicting those outcomes were analyzed.

Results: After median follow-up of 63 (IQR 30-110) months, 35.8\% had GC-dependent asthma and/or ENT manifestations, $12.9 \%$ had $\geq 1$ vasculitis relapse(s), $14.3 \%$ had both phenotypes, $14.6 \%$ were in complete remission, $14.4 \%$ were in partial remission and $7.8 \%$ had not reach remission.

Patients with GC-dependent asthma/ENT manifestations were younger at diagnosis $(p<0.0001)$, had more frequent GC-treated asthma before overt EGPA $(p=0.002)$, had more ENT manifestations $(p=0.01)$ and less frequent MPO-ANCA $(p<0.0001)$. Their daily GC dose was higher at every time point $(p<0.0001)$, and they had more frequent active asthma at last follow-up $(p<0.0001)$.

Patients with vasculitis relapse(s) had more frequently neurological manifestations at diagnosis $(p=0.002)$ and MPO-ANCA positivity $(p<0.0001)$, and less frequently pulmonary infiltrates $(p=0.031)$. Median time from diagnosis-to- $1^{\text {st }}$ vasculitis relapse was $25(11-60)$ months. During follow-up, their daily GC dose was lower than those with GC-dependent asthma/ ENT manifestations, but similar to those in complete remission. At last follow-up, neurological sequelae tended to be more frequent $(p=0.06)$.

Finally, patients in complete remission were older $(p<0.0001)$, had more fever $(p=0.03)$, less GC-treated asthma $(p=0.002)$ and ENT manifestations at diagnosis $(p=0.01)$, lower daily $\mathrm{GC}$ dose during follow-up $(p<0.0001)$, lower eosinophils count at 6 months $(p=0.002)$ and less frequent sequelae $(p=0.003)$

Conclusion: EGPA seems to evolve toward distinct phenotypic profiles, which could be identified using baseline and follow-up characteristics. Early identification of those profiles could allow guided choices of the best therapeutic option.

Disclosure of Interests: Matthias Papo: None declared, Renato A. Sinico: None declared, Vítor Teixeira: None declared, Maria-Letizia Urban: None declared, Juliane Mahrhold: None declared, Sara Monti: None declared, Giulia Cassone: None declared, Franco Schiavon: None declared, Benjamin Seeliger: None declared, Thomas Neumann: None declared, Claus Kroegel: None declared, Matthieu Groh: None declared, Chiara Marvisi: None declared, Maxime Samson: None declared, Thomas Barba: None declared, David Jayne Grant/research support from: David Jayne has received research grants from Chemocentryx, GSK, Roche/Genentech and Sanofi-Genzyme. He has received consultancy fees from Astra-Zeneca, Boehringer-Ingelheim, Chemocentryx, Chugai, GSK, Infla-RX, Insmed and Takeda, Bernhard Hellmich Consultant for: Roche, Speakers bureau: Abbvie, MSD, Roche, Novartis, Pfizer, Carlomaurizio Montecucco Speakers bureau: AbbVie, Bristol-Myers Squibb, Celgene, Sanofi, Genzyme, Lilly, MSD, Pfizer, UCB, Carlo Salvarani Grant/research support from: Roche, Consultant for: Eli Lilly and Company, Roche, Abbvie, Jean-Emmanue Kahn: None declared, Bernard Bonnotte: None declared, Cécile-Audrey Durel: None declared, Luc Mouthon: None declared, Xavier Puéchal: None declared, Loïc Guillevin: None declared, Giacomo Emmi: None declared, Augusto Vaglio: None declared, Benjamin Terrier: None declared

DOI: 10.1136/annrheumdis-2019-eular.4013

\section{SAT0240 DEVELOPMENT AND PRELIMINARY VALIDATION OF THE BEHCYET'S SYNDROME OVERALL DAMAGE INDEX (BODI)}

Matteo Piga ${ }^{1}$, Alberto Floris ${ }^{1}$, Gerard Espinosa ${ }^{2}$, Nikolaos Kougkas ${ }^{3}$, Andrea Lo Monaco $^{4}$, Giuseppe Lopalco ${ }^{5}$, Ida Orlando ${ }^{6}$, Vittorio Pirani ${ }^{\text {, }}$

Ernestina Santos ${ }^{8,9}$, Luísa Serpa Pinto ${ }^{10}$, George Bertsias ${ }^{3}$, Luca Cantarini ${ }^{6}$, Alberto Cauli ${ }^{1}$, Ricard Cervera ${ }^{2}$, João Correia ${ }^{10}$, Marcello Govoni ${ }^{4}$, Florenzo lannone ${ }^{5}$, Ana Martins Da Silva 8,9 , Piergiorgio Neri ${ }^{11}$

Carlos Vasconcelos ${ }^{9}$, Monica Muntoni ${ }^{12}$, Alessandro Mathieu ${ }^{1} .{ }^{1}$ University of Cagliari, Rheumatology, Monserrato, Italy, ${ }^{2}$ University of Barcelona, Autoimmune Diseases, Barcelona, Catalonia, Spain; ${ }^{3}$ University of Crete, Rheumatology, Clinical Immunology and Allergy, Heraklion, Greece; ${ }^{4}$ University of Ferrara, Rheumatology, Ferrara, Italy; ${ }^{5}$ University of Bari, Rheumatology, Bari, Italy; ${ }^{6}$ University of Siena, Rheumatology, Siena, Italy; ${ }^{7}$ Università Politecnica delle Marche, Ophthalmology, Ancona, Italy, ${ }^{8}$ Centro Hospitalar do Porto/Hospital de Santo António, Neurology, Porto, Portugal; ${ }^{9}$ University of Porto, UMIB, Abel Salazar Biomedical Sciences Institute, Porto, Portugal; ${ }^{10}$ Hospital Santo Antonio Centro Hospitalar do Porto, Unidade de Imunologia Clinica, Porto, Portugal;

${ }^{11}$ Cleveland Clinic Abu Dhabi, Eye Institute, Abu Dhabi, United Arab Emirates;

${ }^{12}$ Patient delegate, Cagliari, Italy

Background: Irreversible organ damage is considered a core outcome by the OMERACT working group. However, no specific tools are currently available to detect and measure damage accrual in Behçet's syndrome (BS).

Objectives: To develop and preliminarily validate the Behçet's syndrome Overall Damage Index (BODI). NCT03803462.

Methods: A preliminary version of the instrument (p-BODI) was developed by reviewing pre-existing tools [e.g. Vasculitis damage index (VDI)] and through an extensive literature review. p-BODI was then reviewed and implemented by a multi-rounds Delphi process, involving an international and multidisciplinary (5 rheumatologists, 4 internist, 1 ophthalmologist, 1 neurologist) panel of experts in BS management and a patients' delegate. A group of clinicians (CG), not involved in the BODI development, was asked to independently score a set of clinical vignettes, in order to test the instrument reliability, after a training process consisting of a user manual and a video-tutorial. Then, Cohen's $K$ and Intra-class correlation coefficient (ICC) between assessors and gold standard were calculated. Afterwards, BODI validation was conducted according to the OMERACT Filter 2.0 in a multicenter BS cohort.

Results: Starting from a list of 120 candidate items, the final version of BODI consisted of 4 overarching principles, 30 items and 12 sub-items (each of them scores one point) grouped in 8 domains (figure).

In terms of reliability, the mean $\mathrm{K}$ coefficient was $0.84(95 \% \mathrm{Cl} 0.78$ to $0.90)$ and the ICC was $0.88(95 \% \mathrm{Cl} 0.80-0.95)$.

Validation cohort consisted of 228 BS patients $(49.1 \%$ males), with a median (IQR) age and disease duration of 46.9 (35.5-55.0) and 11.7 (5.8-20.7) years, respectively. Overall, prevalence of any BODI damage (BODI $\geq 1$ ) was $56.1 \%$ with a median score of $1.0(0-2.0)$. In regard of construct validity, BODI score significantly correlated with VDI (Spearman's rho $0.693, p<0.001)$. Besides, BODI score did not correlate with BDCAF (rho-0.016, $\mathrm{p}=0.807$ ), contrary to VDI (rho $0.141, \mathrm{p}=0.034$ ). Such results support the validity of $\mathrm{BODI}$, unlike $\mathrm{VDI}$, in discriminating damage from current disease activity in BS. On multiple regression analysis, factors independently associated to higher BODI damage score were male gender $(\beta$ coefficient $0.143 ; p=0.014)$, longer disease duration $(\beta 0.221 ; p<$ $0.001)$, past major organ involvement $(\beta 0.377 ; p<0.001)$ and required use of anti-TNF $\alpha$ inhibitors $(\beta$ 0.222; $p<0.001$ ).

Full agreement among the $C G$ was reached in judging BODI as a credible, comprehensive, easy to use, timesaving and acceptable instrument. Conclusion: BODI is the first tool specifically developed to assess damage in BS. Preliminary data encourage further validation of BODI in more extended and multi-ethnic BS cohorts before being applied in clinical practice and as a therapeutic outcome. 